\title{
Hour Times Millimole Per Liter Per Milligram Per Gram
}

National Cancer Institute

\section{Source}

National Cancer Institute. Hour Times Millimole Per Liter Per Milligram Per Gram. NCI

Thesaurus. Code C117922.

Hour times millimole per liter, divided by milligram per gram. 